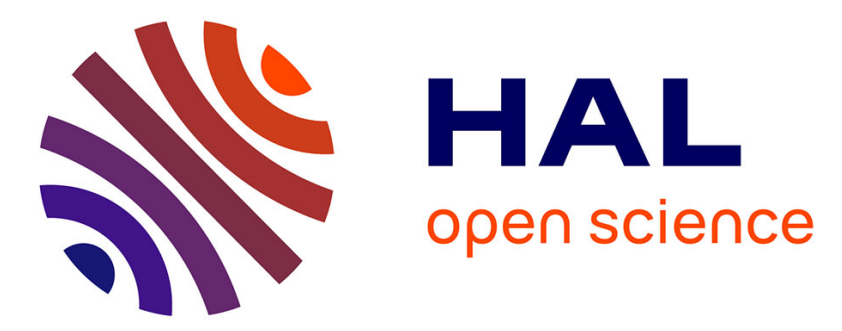

\title{
Three-dimensional elasto-optical interaction for reflectometric detection of diffracted acoustic fields in picosecond ultrasonics
}

Thomas Dehoux, Nikolay Chigarev, Clément Rossignol, Bertrand Audoin

\section{- To cite this version:}

Thomas Dehoux, Nikolay Chigarev, Clément Rossignol, Bertrand Audoin. Three-dimensional elastooptical interaction for reflectometric detection of diffracted acoustic fields in picosecond ultrasonics. Physical Review B: Condensed Matter and Materials Physics (1998-2015), 2007, 76 (2), pp.024311. 10.1103/PhysRevB.76.024311 . hal-01658485

\author{
HAL Id: hal-01658485 \\ https://hal.science/hal-01658485
}

Submitted on 7 Dec 2017

HAL is a multi-disciplinary open access archive for the deposit and dissemination of scientific research documents, whether they are published or not. The documents may come from teaching and research institutions in France or abroad, or from public or private research centers.
L'archive ouverte pluridisciplinaire HAL, est destinée au dépôt et à la diffusion de documents scientifiques de niveau recherche, publiés ou non, émanant des établissements d'enseignement et de recherche français ou étrangers, des laboratoires publics ou privés. 


\title{
Three-dimensional elasto-optical interaction for reflectometric detection of diffracted acoustic fields in picosecond ultrasonics
}

\author{
Thomas Dehoux, Nikolay Chigarev, Clément Rossignol, and Bertrand Audoin* \\ Université Bordeaux 1; CNRS; UMR 5469, Laboratoire de Mécanique Physique, F-33405 Talence, France
}

(Received 19 March 2007; revised manuscript received 15 May 2007; published 31 July 2007)

\begin{abstract}
The three-dimensional (3D) photoelastic interaction involved in the detection mechanism of picosecond ultrasonics is investigated in micrometric metallic films. In pump-probe experiments, the laser source beam is focused to a spot size of less than $1 \mu \mathrm{m}$. A 3D diffracted acoustic field is generated at high frequencies of several tens of gigahertz, containing longitudinal and shear waves altogether. Their propagation changes the dielectric permittivity tensor and the material becomes optically heterogeneous. Consequently, the detection process is modeled through the propagation of the laser probe beam in a material with dielectric properties varying in all directions. Thus, the solution of Maxwell's equations leads to a differential system, the source term of which is proportional to the acoustic field itself. In the frame of small perturbation theory, the latter is decomposed into a continuous sum of monochromatic plane waves. The scattered electromagnetic field is described using the matricant, and the ensuing analytical solution then allows analyzing the 3D photoelastic interaction. The contribution of acoustic diffraction and shear wave detection to the reflectometric signal is put into relief. Good agreement with experiments performed in a $1 \mu \mathrm{m}$ thick aluminum film is found.
\end{abstract}

DOI: 10.1103/PhysRevB.76.024311

PACS number(s): 78.20.Hp, 43.35.+d, 46.40.Cd, 78.47.+p

\section{INTRODUCTION}

Since the early works of Thomsen $e t$ al. in the middle of the $1980 \mathrm{~s},{ }^{1,2}$ the potentialities of picosecond ultrasonics have been enlarged. This technique allows the noncontact generation and detection of sound pulses at frequencies of several tens of gigahertz. Owing to their small wavelengths, the propagation of these high frequency elastic waves permits evaluation of mechanical, optical, and thermal properties of nanometric structures.

Pump-probe experiment is a widespread optical method based on the use of two laser pulses, one for generation and the other for detection. The absorption of the pump laser pulse, the duration of which is a few femtoseconds, creates a thermal stress through thermoelastic coupling. ${ }^{3}$ As a result, the sudden volume expansion launches an acoustic wave which propagates into the medium. Until recently, only longitudinal plane waves could be generated. However, for some applications, such as the measurement of the sample elasticity, transverse acoustic waves are of great interest. Their generation has been first achieved by mode conversion of longitudinal plane waves at the interface of polycrystalline films deposited on anisotropic substrate with broken symmetry axis. Their detection was performed, through the interaction of the probe pulse with the acoustic waves, by reflectometric ${ }^{4}$ or interferometric ${ }^{5}$ measurements. Lately, pumping through a transparent layer directly inside the anisotropic medium with tilted axes led to a more efficient way to obtain plane transverse waves. ${ }^{6,7}$

Over the years, several laser techniques were developed for surface wave detection. ${ }^{8-10}$ Recently, improving the focusing of the pump beam allowed the imaging of high frequency surface waves. ${ }^{11,12}$ Besides, bulk wave diffraction effects were also studied in submillimetric samples by focusing the laser beam through a microscope objective in order to reduce the lateral extent of the source. The far field limit was reached by propagating the acoustic pulse on long distances at low temperature to diminish acoustic attenuation. ${ }^{13,14}$ Lately, increasing the magnification of the microscope objective, in order to focus the laser beam to a smaller spot on the surface, has allowed shear wave observation by interferometric measurements in submicrometric films. ${ }^{15}$

In strongly absorbing materials, the probe light is almost entirely reflected at the free surface, and only its phase is modified by the surface displacement. Thus, to describe interferometric signals in opaque materials, only the displacement is required, ${ }^{16}$ and one can solve the equation of motion for a three-dimensional geometry, without considering any interaction with the probe beam. ${ }^{15,17,18}$ As the optical penetration increases, the probe beam senses the in-depth optical index variation due to the strain propagation. Both phase and amplitude of the reflected light are affected. To model interferometric measurements in quasitransparent media, the interaction between the laser probe beam and the threedimensional acoustic field must be investigated. On the other hand, considering reflectometric measurements in any material, whatever its optical penetration may be, this acoustooptic coupling must be taken into account.

In the present paper, a semianalytical model is developed to investigate the physical processes involved in the picosecond ultrasonics detection. In transversally isotropic media, the response to a laser point source can be deduced from the response to a line source focused on the surface of the material. ${ }^{19}$ Thus, in this work, the interaction of the laser probe beam with a two-dimensional acoustic field, generated by a laser line source with small lateral width, is firstly modeled. Then, the results are extended analytically to account for the three-dimensional (3D) geometry of the laser point source.

The strain field, while propagating, changes the optical properties in all directions through an elasto-optic coupling phenomenon. ${ }^{20}$ The material, therefore, becomes optically heterogeneous and, to describe the electromagnetic propaga- 
tion, Maxwell's equations must be solved. This formulation leads to a second-order partial differential system with coefficients varying in two directions. The variation of optical properties is linearized in one direction with the perturbation theory in order to apply a spatial Fourier transform. Thereby, the acoustic field is decomposed into a sum of plane waves. Assuming the linearity of the acousto-optic coupling, the core of the calculation is the oblique interaction of the probe beam with only one acoustic plane wave, with no regard to any interface.

In picosecond ultrasonics, a matrix formalism has already been developed to describe the oblique incidence of the probe laser beam on a plane longitudinal wave. ${ }^{21}$ Recently, by introducing the contribution of plane shear waves through the photoelastic tensor, the differential equations ${ }^{22}$ were solved using Green's functions. ${ }^{23}$ In the present paper, by taking into account acoustic diffraction, these calculations are extended using the matricant. ${ }^{24,25}$ This mathematical tool is suited to solve first-order ordinary differential systems, the coefficients of which may be varying. Thus, the optical diffraction by a medium with continuously varying properties is calculated analytically, in the time and space Fourier domains, for a line source.

Through the detection of the scattered optical field, the mechanisms of the three-dimensional elasto-optic interaction are analyzed. We thereby report on the effects of acoustic diffraction on the picosecond ultrasonics signal, notably through the influence of shear waves. By using a classical pump-probe experimental setup, ${ }^{26}$ reflectometric measurements are performed. The calculated signals are then compared with the experimental data to study the propagation of diffracted longitudinal and transverse acoustic pulses, and to put into relief the effects of acoustic diffraction.

\section{ELASTICALLY INDUCED TRANSIENT OPTICAL HETEROGENEITY}

The dominant generation phenomenon in low-intensity radiation interaction with metals is the thermoelastic process. ${ }^{2,3}$ The pump laser beam penetrates into the material as the electromagnetic field is absorbed, and the whole illuminated area acts as a spatiotemporal heat distribution. The latter diffuses inside the material, and, neglecting the electronic evolutions which occur in metals, ${ }^{27}$ the temperature behavior is ruled by the Fourier equation of heat diffusion. ${ }^{28}$ The thermal energy is converted into mechanical energy through a volume dilatation of the sample. A thermoelastic stress, proportional to the gradient of the temperature, is created, and the propagation of the acoustic displacement is described through the equation of motion. ${ }^{18}$

The response to a laser point source can be expressed in the space domain as the Hankel transform of the Fourier spectrum of the response to a line source. It is equivalent to the continuous sum of the responses to line sources with different orientations on the surface of the material. ${ }^{19}$ Considering transversally isotropic media, the axisymmetry of the physical properties allows calculating the response to only one line source. Hence, the equations describing the generation process are written for a laser line source in Car- tesian coordinates $\left(x_{1}, x_{2}, x_{3}\right)$. A free isotropic film, the thickness of which is along $\boldsymbol{x}_{1}$, is modeled. The acoustic waves are generated by the absorption of radiation of the incident pump laser beam, focused on the surface along a line in the direction $x_{3}$. Due to the symmetry of this configuration, the acoustic and electromagnetic fields do not depend on $x_{3}$.

To obtain ordinary differential equations, a spatiotemporal Fourier transform is applied in the $\left(x_{2}, t\right)$ space. This mathematical operation will be denoted by the grapheme tilde over the letter, and the associated dual space will be referred to as $\left(k_{2}, \omega\right)$. Thereby, the analytical expression of the spectrum of the displacement is obtained. The expression of the strain $^{(2)} \tilde{\bar{\eta}}$ is finally found, calculating the symmetrical gradient of the displacement. Using Auld's contracted notation, ${ }^{29}$ the strain tensor is expressed in its matrix form, for the geometry previously described:

$$
\left[\widetilde{\eta}\left(x_{1}, k_{2}, \omega\right)\right]=\left[\begin{array}{ccc}
\tilde{\eta}_{1} & \tilde{\eta}_{6} & 0 \\
\tilde{\eta}_{6} & \tilde{\eta}_{2} & 0 \\
0 & 0 & 0
\end{array}\right] .
$$

When the optical penetration depth is smaller than the acoustic wavelength, the probe beam is mainly sensitive to the displacement of the surface. The amplitude of the reflected light is barely affected, but its phase is significantly modified. In this case, the interferometric response of an opaque material can be represented by the normal displacement of the free surface of the medium in the direction $x_{1},{ }^{15}$ and the acousto-optic interaction can be neglected. However, if the absorption is weak, the probe beam is sensitive to the modulation of optical properties created by the in-depth strain ${ }^{(2)} \bar{\eta}$. Part of the light penetrates into the material and is backscattered by the transient change of optical index induced by the acoustic propagation. Thus, optical interferences, which affect both phase and amplitude of the scattered light, arise. This phenomenon appears in the reflectometric signal, as well as in the interferometric response of weakly absorbing materials through Brillouin oscillations. ${ }^{2}$ Therefore, the interaction of the light beam with the diffracted acoustic field must be taken into account to represent these detection mechanisms.

This detection process has been fully described for onedimensional problems. ${ }^{1,16}$ Nevertheless, in the case of point generation and point detection, when pump and probe are at the same position, experimental signals have demonstrated the existence of shear waves in polycrystalline aluminum. ${ }^{15}$ Yet, the latter cannot be generated directly by a thermoelastic point source in an infinite isotropic material. The presence of the surface allows launching directly of shear waves T. However, for symmetry reasons, they can propagate in any direction but $k_{2}=0$. Then, when reaching the other interface, to satisfy the laws of refraction for a given $k_{2} \neq 0$, they give birth to reflected shear waves $2 \mathrm{~T}$ and mode converted longitudinal waves TL. In the same way, longitudinal waves $\mathrm{L}$ are reflected into $2 \mathrm{~L}$ and converted into LT waves when $k_{2} \neq 0$. Since these contributions LT and TL only travel in oblique directions defined by $k_{2} \neq 0$, they scatter light in directions different from the incident one. Therefore, the acoustic diffraction, created by the finite size of the source, imposes to 
study the two-dimensional interaction of the normal incidence probe beam with the acoustic field.

The change of the optical properties of the sample, due to propagation of the elastic deformation inside the bulk, is described through the linear acousto-optic interaction. ${ }^{20}$ The variation ${ }^{(2)} \bar{\varepsilon}^{s}$ of the dielectric tensor ${ }^{(2)} \bar{\varepsilon}$ is related to the strain by the fourth-order photoelastic tensor ${ }^{(4)} \bar{P}$ through the equation ${ }^{(2)} \bar{\varepsilon}^{s}={ }^{(4)} \bar{P}:{ }^{(2)} \bar{\eta}$. For isotropic materials, the following matrix form is obtained:

$$
\left[\varepsilon\left(x_{1}, x_{2}, t\right)\right]=\varepsilon^{h}[I]+\left[\varepsilon^{s}\left(x_{1}, x_{2}, t\right)\right],
$$

with

$$
\begin{aligned}
{\left[\varepsilon^{s}\left(x_{1}, x_{2}, t\right)\right] } & \\
\quad & =\left[\begin{array}{ccc}
P_{11} \eta_{1}+P_{12} \eta_{2} & P_{66} \eta_{6} & 0 \\
P_{66} \eta_{6} & P_{12} \eta_{1}+P_{11} \eta_{2} & 0 \\
0 & 0 & P_{12}\left(\eta_{1}+\eta_{2}\right)
\end{array}\right] \\
& =\left[\begin{array}{ccc}
\varepsilon_{1}^{s} & \varepsilon_{6}^{s} & 0 \\
\varepsilon_{6}^{s} & \varepsilon_{2}^{s} & 0 \\
0 & 0 & \varepsilon_{3}^{s}
\end{array}\right] .
\end{aligned}
$$

In the absence of any acoustic perturbation, the dielectric matrix is given by its homogeneous component $\varepsilon^{h}[I]$. Due to longitudinal strain components $\eta_{1}$ and $\eta_{2}$, the diagonal elements of $[\varepsilon]$ are no longer equal. The surface of wave normals, ${ }^{22}$ defining the optical phase velocity as a function of space, is deformed into an ellipsoid. The off-diagonal part, arising from the shear strain $\eta_{6}$, changes the optical principal axes of the material, and it rotates the surface of wave normals around the axis $\boldsymbol{x}_{3}$. These changes are spatially and temporally modulated by the propagation of the elastic wave, and the material is made continuously heterogeneous.

\section{OBLIQUE LASER INTERACTION WITH A MONOCHROMATIC ACOUSTIC HETEROGENEITY}

The perturbed dielectric tensor (2) is now introduced in Maxwell's equations to describe the optical propagation in a heterogeneous material. Optical plane wave solutions are then proscribed, and a differential system, with space depending coefficients, must be considered. A spatiotemporal Fourier transform in $x_{2}$ and $t$ was applied in the previous section. This mathematical operation is considered as a decomposition of the acoustic field into a continuous sum of monochromatic plane waves propagating in the direction defined by the wave vector $\boldsymbol{k}^{e}=\left(k_{1}^{e}, k_{2}\right)$. The interaction of the normal incidence probe beam with this sum of acoustic plane waves is now formalized in the dual space $\left(k_{2}, \omega\right)$.

Harmonic solutions at angular frequency $\omega$ for the electric and magnetic fields $\boldsymbol{E}$ and $\boldsymbol{H}$ are sought, applying a time Fourier transform. In the remainder, this operation will not be indicated explicitly, and the grapheme tilde will only denote the double Fourier transform in $\left(x_{2}, t\right)$. In the same way, the dependency on $\omega$ will not be pointed out explicitly, since it is not a differential variable. The propagation of the elec- tric field inside a heterogeneous material is then described by Maxwell's equations: $:^{22}$

$$
\begin{aligned}
& \nabla \wedge \boldsymbol{H}\left(x_{1}, x_{2}\right)=-j k_{0}{ }^{(2)} \bar{\varepsilon}\left(x_{1}, x_{2}\right) \boldsymbol{E}\left(x_{1}, x_{2}\right), \\
& \nabla \wedge \boldsymbol{E}\left(x_{1}, x_{2}\right)=j k_{0} \mu \boldsymbol{H}\left(x_{1}, x_{2}\right),
\end{aligned}
$$

where $k_{0}$ is the wave vector in vacuum. In view of amagnetic materials, the magnetic permittivity $\mu$ is a scalar constant. Considering separately the fields $\boldsymbol{E}$ and $\boldsymbol{H}$ leads to a firstorder differential system. When the spatial Fourier transform is applied in the direction $\boldsymbol{x}_{2}$, a convolution in the spectral domain arises from the product ${ }^{(2)} \overline{\boldsymbol{E}} \boldsymbol{E}$. It defines the sum of the interactions of any component of the optical spectrum with each component of the elastic spectrum. In order to analytically solve the differential system in the Fourier domain, the convolution term must be suppressed. To do so, the homogeneous and scattered electromagnetic fields are separated, similarly to the dielectric matrix counterparts in Eq. (2):

$$
\begin{gathered}
\boldsymbol{E}\left(x_{1}, x_{2}\right)=\boldsymbol{E}^{h}\left(x_{1}\right)+\boldsymbol{E}^{s}\left(x_{1}, x_{2}\right), \\
\boldsymbol{H}\left(x_{1}, x_{2}\right)=\boldsymbol{H}^{h}\left(x_{1}\right)+\boldsymbol{H}^{s}\left(x_{1}, x_{2}\right),
\end{gathered}
$$

with $\left\{\boldsymbol{E}^{h}\left(x_{1}\right), \boldsymbol{H}^{h}\left(x_{1}\right)\right\}$ denoting the electromagnetic fields propagating in the homogeneous material along the direction $\boldsymbol{x}_{1}$, and $\left\{\boldsymbol{E}^{s}\left(x_{1}, x_{2}\right), \boldsymbol{H}^{s}\left(x_{1}, x_{2}\right)\right\}$ standing for the scattered fields.

In the frame of linear acoustics, the amplitude of the elastic strain remains small $\left(\eta_{i} \ll 1\right)$, and the formalism of the problem can be simplified by applying the perturbation theory. ${ }^{29}$ The perturbed optical fields $\left\{\boldsymbol{E}^{s}, \boldsymbol{H}^{s}\right\}$ and the perturbation $\left[\varepsilon^{s}\right]$ of the dielectric tensor, given by Eq. (2), are of the same order of magnitude as the acoustic field. A Taylor series expansion is performed to the first order to linearize Eqs. (4):

$$
\begin{aligned}
\nabla \wedge \boldsymbol{E}^{h}\left(x_{1}\right)-j k_{0} \mu \boldsymbol{H}^{h}\left(x_{1}\right) & =0, \\
\nabla \wedge \boldsymbol{H}^{h}\left(x_{1}\right)+j k_{0} \varepsilon^{h} \boldsymbol{E}^{h}\left(x_{1}\right) & =0, \\
\nabla \wedge \boldsymbol{E}^{s}\left(x_{1}, x_{2}\right)-j k_{0} \mu \boldsymbol{H}^{s}\left(x_{1}, x_{2}\right) & =0, \\
\nabla \wedge \boldsymbol{H}^{s}\left(x_{1}, x_{2}\right)+j k_{0} \varepsilon^{h} \boldsymbol{E}^{s}\left(x_{1}, x_{2}\right) & =-j k_{0}\left[\varepsilon^{s}\left(x_{1}, x_{2}\right)\right] \boldsymbol{E}^{h}\left(x_{1}\right) .
\end{aligned}
$$

The left-hand side of this system describes the spatial distribution of the propagative solution, whereas the right-hand side determines its amplitude. As only the source term depends on $\left[\varepsilon^{s}\right]$ under the first-order assumption, the norm of the optical wave vectors is not affected by the variation of optical properties; only the amplitude and the direction of propagation of the optical field are. Thus the optical field is scattered in space, but its frequency remains the same.

Linearizing the two-dimensional elasto-optic interaction has suppressed the product of two $x_{2}$ dependent functions

${ }^{(2)} \overline{\boldsymbol{\varepsilon}}^{s} \boldsymbol{E}$ by neglecting the second-order terms in Eq. (4). The spatial Fourier transform can now be applied merely to Eqs. (6), and no convolution term appears anymore. This 
assumption means that the optical wave diffracted in a direction with a $k_{2}$ component by each acoustic plane wave does not interact with the other acoustic plane waves. Hence, each acoustic plane wave can be studied separately. In a manner similar to the formalism described by Miklós and Lörincz, ${ }^{21}$ the following first-order differential system is found in the Fourier domain $\left(k_{2}, \omega\right)$ from Eqs. (6):

$$
\begin{gathered}
\frac{\tilde{f}^{h}\left(x_{1}\right)}{\partial x_{1}}-\left[A^{h}\right] \widetilde{\boldsymbol{f}}^{h}\left(x_{1}\right)=0, \\
\frac{\tilde{\boldsymbol{f}}^{s}\left(x_{1}, k_{2}\right)}{\partial x_{1}}-\left[\widetilde{A}^{s}\left(k_{2}\right)\right] \widetilde{\boldsymbol{f}}^{s}\left(x_{1}, k_{2}\right)=\left[\widetilde{a}\left(x_{1}, k_{2}\right) \tilde{\mathrm{f}}^{h}\left(x_{1}\right),\right.
\end{gathered}
$$

with

$$
\begin{aligned}
& \widetilde{\boldsymbol{f}}^{h}\left(x_{1}\right)=\left\{\begin{array}{c}
\widetilde{E}_{3}^{h} \\
\widetilde{H}_{2}^{h} \\
\widetilde{E}_{2}^{h} \\
\widetilde{H}_{3}^{h}
\end{array}\right\}, \quad \widetilde{\boldsymbol{f}}^{s}\left(x_{1}, k_{2}\right)=\left\{\begin{array}{c}
\widetilde{E}_{3}^{s} \\
\widetilde{H}_{2}^{s} \\
\widetilde{E}_{2}^{s} \\
\widetilde{H}_{3}^{s}
\end{array}\right\}, \\
& {\left[A^{h}\right]=j k_{0}\left[\begin{array}{cc|cc}
0 & \mu & 0 & 0 \\
\varepsilon^{h} & 0 & 0 & 0 \\
\hline 0 & 0 & 0 & -\mu \\
0 & 0 & -\varepsilon^{h} & 0
\end{array}\right]=\left[\begin{array}{cc}
{\left[A_{3}^{h}\right]} & 0 \\
0 & {\left[A_{2}^{h}\right]}
\end{array}\right] \text {, }} \\
& {\left[\widetilde{A}^{s}\right]=j k_{0}\left[\begin{array}{cc|cc}
0 & \mu & 0 & 0 \\
\varepsilon^{h}-k_{2}^{2} / k_{0}^{2} \mu & 0 & 0 & 0 \\
\hline 0 & 0 & 0 & k_{2}^{2} / k_{0}^{2} \varepsilon^{h}-\mu \\
0 & 0 & -\varepsilon^{h} & 0
\end{array}\right]} \\
& =\left[\begin{array}{cc}
{\left[\widetilde{A}_{3}^{s}\right]} & 0 \\
0 & {\left[\widetilde{A}_{2}^{s}\right]}
\end{array}\right] \text {, } \\
& {[\widetilde{a}]=j k_{0}\left[\begin{array}{cc|cc}
0 & 0 & 0 & 0 \\
\widetilde{\varepsilon}_{3}^{s} & 0 & 0 & 0 \\
\hline 0 & 0 & -k_{2} \widetilde{\varepsilon}_{6}^{s} / k_{0} \varepsilon^{h} & 0 \\
0 & 0 & -\widetilde{\varepsilon}_{2}^{s} & 0
\end{array}\right]} \\
& =j k_{0}\left[\begin{array}{cc|cc}
0 & 0 & 0 & 0 \\
\tilde{a}_{21} & 0 & 0 & 0 \\
\hline 0 & 0 & \tilde{a}_{33} & 0 \\
0 & 0 & \tilde{a}_{43} & 0
\end{array}\right]=\left[\begin{array}{cc}
{\left[\tilde{a}_{3}\right]} & 0 \\
0 & {\left[\tilde{a}_{2}\right]}
\end{array}\right] \text {, }
\end{aligned}
$$

where $[\tilde{a}]$ contains the change of optical properties.

The upper-left $2 \times 2$ matrices $\left[A_{3}^{h}\right],\left[\widetilde{A}_{3}^{s}\right]$, and $\left[\tilde{a}_{3}\right]$ represent the propagation of the optical mode polarized along the axis $\boldsymbol{x}_{3}$, whereas the lower-right $2 \times 2$ matrices $\left[A_{2}^{h}\right],\left[\tilde{A}_{2}^{s}\right]$, and $\left[\widetilde{a}_{2}\right]$ stand for the polarization in the plane $\left(\boldsymbol{x}_{1}, \boldsymbol{x}_{2}\right)$. As the other submatrices are null, there is no coupling between these two modes, and no rotation of the scattered polarization with respect to the incident one is to be expected. The upper-left matrix system will be denoted with the index $p=3$, and the lower-right system with $p=2$. Thus, the solution of the problem is split to study separately the influence of each polarization $p$. The system (7) is then rewritten to manipulate smaller $2 \times 2$ matrices:

$$
\begin{gathered}
\frac{\widetilde{\partial f}_{p}^{h}\left(x_{1}\right)}{\partial x_{1}}-\left[A_{p}^{h} \widetilde{\boldsymbol{f}}_{p}^{h}\left(x_{1}\right)=0,\right. \\
\frac{\partial \tilde{\boldsymbol{f}}_{p}^{s}\left(x_{1}, k_{2}\right)}{\partial x_{1}}-\left[\widetilde{A}_{p}^{s}\left(k_{2}\right)\right] \tilde{f}_{p}^{s}\left(x_{1}, k_{2}\right)=\left[\widetilde{a}_{p}\left(x_{1}, k_{2}\right)\right] \widetilde{\boldsymbol{f}}_{p}^{h}\left(x_{1}\right),
\end{gathered}
$$

with

$$
\tilde{\boldsymbol{f}}_{p}^{q}=\left\{\begin{array}{c}
\widetilde{E}_{p}^{q} \\
\tilde{H}_{5-p}^{q}
\end{array}\right\},
$$

where the index $q=h$ and $s$ stands for the homogeneous and scattered solutions, respectively.

\section{SOLUTION USING THE MATRICANT}

The determination of the matrix named matricant, which defines the solution at any position $x_{1}$ as a function of the boundary conditions expressed in $x_{0}$, allows analytically solving first-order ordinary differential systems, the coefficients of which may be a function of the chosen variable $x_{1} .{ }^{24}$ This technique is suitable for the study of the propagation in media with properties varying in one direction. Hence, the diffraction on inhomogeneities represented by a continuous or discrete function can be analyzed. ${ }^{25}$ Multilayered structures could also be examined by defining the succession of layers as a discrete function of the concerned properties, without having to specify explicitly the boundary conditions at each interface.

The differential system (12) is now solved using the matricant to determine the homogeneous and scattered fields $\boldsymbol{f}_{p}^{q}$ for each polarization $p$. As the dimension of the system (12) equals 2 , it possesses two independent solutions ${ }^{i} f_{p}^{q}\left(x_{1}, k_{2}\right)$, $i=1,2$. From these, one may construct the integral matrix of the system $X_{p}^{q}\left(x_{1}, k_{2}\right)=\left[{ }^{1} \boldsymbol{f}_{p}^{q}\left(x_{1}, k_{2}\right),{ }^{2} \boldsymbol{f}_{p}^{q}\left(x_{1}, k_{2}\right)\right]$. Introducing a boundary condition located in $x_{1}=x_{0}, X_{p}^{q}\left(x_{1}, k_{2}\right)$ becomes the matricant $\left[\tilde{\mathcal{M}}_{p}^{q}\left(x_{1}, x_{0}, k_{2}\right)\right]$, written for each $p, q$ matrix system as follows:

$$
\begin{aligned}
\frac{\partial\left[\tilde{\mathcal{M}}_{p}^{q}\left(x_{1}, x_{0}, k_{2}\right)\right]}{\partial x_{1}} & =\left[\tilde{A}_{p}^{q}\left(k_{2}\right)\right]\left[\tilde{\mathcal{M}}_{p}^{q}\left(x_{1}, x_{0}, k_{2}\right)\right], \\
{\left[\tilde{\mathcal{M}}_{p}^{q}\left(x_{0}, x_{0}, k_{2}\right)\right] } & =[I] .
\end{aligned}
$$

Using this formalism, as $\left[A_{p}^{q}\right]$ do not depend on $x_{1}$, the matricants $\left[\tilde{\mathcal{M}}_{p}^{q}\right]$ are expressed as exponentials of $\left[A_{p}^{q}\right]$. They are calculated by diagonalizing $\left[A_{p}^{q}\right]$ to obtain exponentials of 
its eigenvalues, which are actually the optical wave numbers. This step is equivalent to finding the optical dispersion equation, and fixing the origin of the axis $\boldsymbol{x}_{1}$ at $x_{0}=0$, the matrices $\left[\mathcal{M}_{p}^{q}\right]$ are expressed as follows:

$$
\begin{aligned}
& {\left[\tilde{\mathcal{M}}_{p}^{q}\left(x_{1}, x_{0}, k_{2}\right)\right]} \\
& \quad=\left[\begin{array}{cc}
\left(e^{j k_{1}^{q} x_{1}}+e^{-j k_{1}^{q} x_{1}}\right) / 2 & \left(e^{j k_{1}^{q} x_{1}}-e^{-j k_{1}^{q} x_{1}}\right) / 2 \beta_{p}^{q} \\
\beta_{p}^{q}\left(e^{j k_{1}^{q} x_{1}}-e^{-j k_{1}^{q} x_{1}}\right) / 2 & \left(e^{j k_{1}^{q} x_{1}}+e^{-j k_{1}^{q} x_{1}}\right) / 2
\end{array}\right],
\end{aligned}
$$

with $k_{1}^{h}=k_{0} n, k_{1}^{s}=\left(k_{0}^{2} n^{2}-k_{2}^{2}\right)^{1 / 2}, \beta_{3}^{q}=k_{1}^{q} / k_{0} \mu, \beta_{2}^{q}=-k_{0} \varepsilon / k_{1}^{q}$, and $n$ being the refractive index of the material. Owing to the first-order linearization of Eq. (4), the projection $k_{1}^{q}$ of the optical wave vector in the direction $\boldsymbol{x}_{1}$ corresponds to the ordinary wave vector since the material is initially isotropic. Expression (15) shows that the matricant $\left[\mathcal{M}_{p}^{q}\right]$ connects the field at any position $x_{1}$ to the boundary condition at $x_{0}$ for the $p$ polarization of the $q$ field, and it can be seen as a propagation matrix. The second equation in Eq. (14) normalizes the matricants to preserve the amplitude of the solutions. The scattered electromagnetic field, solution of Eq. (12), can then be written as follows:

$$
\begin{aligned}
\tilde{\boldsymbol{f}}_{p}^{s}\left(x_{1}, k_{2}\right)= & {\left[\tilde{\mathcal{M}}_{p}^{s}\left(x_{1}, x_{0}, k_{2}\right)\right]\left[\tilde{m}\left(x_{1}, k_{2}\right)\right] f_{p}^{h}\left(x_{0}\right) } \\
& +\left[\tilde{\mathcal{M}}_{p}^{s}\left(x_{1}, x_{0}, k_{2}\right)\right] \tilde{\boldsymbol{f}}_{p}^{s}\left(x_{0}, k_{2}\right),
\end{aligned}
$$

with

$$
\begin{aligned}
{\left[\tilde{m}\left(x_{1}, k_{2}\right)\right]=} & \int_{x_{0}}^{x_{1}}\left[\tilde{\mathcal{M}}_{p}^{s}\left(x_{1}^{\prime}, x_{0}, k_{2}\right)\right]^{-1}\left[\widetilde{a}_{p}\left(x_{1}^{\prime}, k_{2}\right)\right] \\
& \times\left[\mathcal{M}_{p}^{h}\left(x_{1}^{\prime}, x_{0}\right)\right] d x_{1}^{\prime},
\end{aligned}
$$

the coefficients of which are given in the Appendix. The first and second terms of the right-hand side of expression (16) represent the propagation of the source term of Eq. (12) and of the boundary condition, respectively. Thus, expression (16) will be calculated explicitly in the next section by applying boundary conditions. The optical field scattered by the sum of acoustic plane waves will then be obtained by applying an inverse spatial Fourier transform.

\section{OPTICAL DETECTION OF ACOUSTIC DIFFRACTION}

The incident electric field $\boldsymbol{E}^{i n c} e^{j k_{0} x_{1}}$ propagates in vacuum in the direction $\boldsymbol{x}_{1}$, with a wave number $k_{0}$. Part of its energy is reflected back at the vacuum-solid interface in the direction $-x_{1}$, with a reflection coefficient $\left[r^{h}\right]$. The superposition of these two contributions defines the homogeneous fields in vacuum ${ }^{v} \boldsymbol{E}^{h}$ and ${ }^{v} \boldsymbol{H}^{h}$. Their $p$ components are written as follows, the quantities denoted with the upper-left index $v$ being expressed in vacuum $(n=1)$ :

$$
\begin{gathered}
{ }^{v} \widetilde{E}_{p}^{h}\left(x_{1}, k_{2}\right)=\left(e^{j k_{0} x_{1}}+r_{p}^{h} e^{-j k_{0} x_{1}}\right) E_{p}^{i n c}, \\
{ }^{v} \widetilde{H}_{5-p}^{h}\left(x_{1}, k_{2}\right)={ }^{v} \beta_{p}^{h}\left(e^{j k_{0} x_{1}}-r_{p}^{h} e^{-j k_{0} x_{1}}\right) E_{p}^{i n c},
\end{gathered}
$$

with ${ }^{v} \beta_{p}^{h}=(-1)^{p-1}$. The $E_{p}^{i n c}$ are the amplitude components of the incident field in the direction $p$.

The transmitted energy then penetrates inside the material. It is backscattered by the in-depth strain, with a coefficient $\left[r^{s}\right]$ which depends on space. This diffracted field crosses the interface in the direction $-\boldsymbol{x}_{1}$. Thereby, the scattered fields ${ }^{v} \boldsymbol{E}^{s}$ and ${ }^{v} \boldsymbol{H}^{s}$, propagating in vacuum, are expressed as

$$
\begin{aligned}
{ }^{v} \widetilde{E}_{p}^{s}\left(x_{1}, k_{2}\right) & =\widetilde{r}_{p}^{s}\left(k_{2}\right) E_{p}^{i n c} e^{-j k_{0} x_{1}}, \\
{ }^{v} \widetilde{H}_{5-p}^{s}\left(x_{1}, k_{2}\right) & =-{ }^{v} \beta_{p}^{s} \widetilde{r}_{p}^{s}\left(k_{2}\right) E_{p}^{i n c} e^{-j k_{0} x_{1},},
\end{aligned}
$$

where ${ }^{v} \beta_{p}^{s}=(-1)^{p-1}\left({ }^{v} k_{1}^{s} / k_{0}\right)^{2 p-5}$ and ${ }^{v} k_{1}^{s}=\left(k_{0}^{2}-k_{2}^{2}\right)^{1 / 2}$. The expression of $\left[\tilde{r}^{s}\right]$, defining the amplitude and phase of the scattered field $\boldsymbol{f}^{s}$, is calculated by applying boundary conditions. The continuity of the electromagnetic fields at $x_{1}=x_{0}$ $=0$ yields eight equations:

$$
\begin{aligned}
\widetilde{E}_{p}^{h}\left(x_{0}\right) & =\left(1+r_{p}^{h}\right) E_{p}^{i n c}, \\
\widetilde{H}_{5-p}^{h}\left(x_{0}\right) & =\left[{ }^{v} \beta_{p}^{h}\left(1-r_{p}^{h}\right)\right] E_{p}^{i n c}, \\
\widetilde{E}_{p}^{s}\left(x_{0}, k_{2}\right) & =\widetilde{r}_{p}^{s} E_{p}^{i n c},
\end{aligned}
$$

$$
\widetilde{H}_{5-p}^{s}\left(x_{0}, k_{2}\right)=-{ }^{v} \beta_{p}^{s} \widetilde{r}_{p}^{s} E_{p}^{i n c} .
$$

As there are ten unknowns, $\widetilde{E}_{p}^{h}\left(x_{0}\right), \widetilde{H}_{5-p}^{h}\left(x_{0}\right), \widetilde{E}_{p}^{s}\left(x_{0}, k_{2}\right)$, $\widetilde{H}_{5-p}^{s}\left(x_{0}, k_{2}\right)$, and $\widetilde{r}_{p}^{s}$, two more equations are needed. They are provided by the addition of a virtual interface. It is assumed that, after a certain distance $x_{1}^{h}$, the material is homogeneous and only outgoing waves are propagating. The boundary condition (20) is propagated up to $x_{1}^{h}$ according to Eq. (16), and nullifying the amplitude of the terms in $\exp \left(-j k_{1}^{s} x_{1}^{h}\right)$ leads to

$$
\begin{aligned}
& {\left[\tilde{m}_{7-2 p, 7-2 p}\left(x_{1}^{h}, k_{2}\right)-\frac{\tilde{m}_{8-2 p, 7-2 p}\left(x_{1}^{h}, k_{2}\right)}{\beta_{p}^{s}}\right] E_{p}^{h}\left(x_{0}\right)} \\
& +\left[\tilde{m}_{7-2 p, 8-2 p}\left(x_{1}^{h}, k_{2}\right)-\frac{\widetilde{m}_{8-2 p, 8-2 p}\left(x_{1}^{h}, k_{2}\right)}{\beta_{p}^{s}}\right] H_{5-p}^{h}\left(x_{0}\right) \\
& \quad=-\widetilde{E}_{p}^{s}\left(x_{0}, k_{2}\right)+\frac{1}{\beta_{p}^{s}} \tilde{H}_{5-p}^{s}\left(x_{0}, k_{2}\right) .
\end{aligned}
$$

If the optical penetration depth, which limits the probed area, would be greater than the film thickness $d$, the laser beam would sense the back surface of the film and would be partialy reflected from this interface. Consequently, in the frame of this model, $x_{1}^{h}$ must be less than $d$. The addition of these two equations (21), for $p=1,2$, allows solving the system. Introducing Eq. (20) into Eq. (21), it is found that 


$$
\begin{aligned}
\tilde{r}_{p}^{s}\left(k_{2}\right)= & \frac{t_{p}^{h} t_{p}^{s}\left(k_{2}\right)}{2}\left[\left(\frac{\tilde{m}_{8-2 p, 8-2 p}}{\beta_{p}^{s}}-\tilde{m}_{7-2 p, 8-2 p}\right) v \beta_{p}^{h}+\frac{\tilde{m}_{8-2 p, 7-2 p}}{\beta_{p}^{s}}\right. \\
& \left.-\tilde{m}_{7-2 p, 7-2 p}\right]
\end{aligned}
$$

with $t_{p}^{h}$ and $t_{p}^{s}\left(k_{2}\right)$ standing for the transmission coefficients from vacuum to medium at normal incidence and from medium to vacuum at the scattered angle, respectively. Then, replacing $m_{i j}$ by their expressions given in the Appendix, the following are obtained:

$$
\begin{gathered}
\tilde{r}_{3}^{s}\left(k_{2}, \omega\right)=j k_{0} \frac{t_{3}^{h} t_{3}^{s}}{2 \beta_{3}^{s}} \int_{0}^{x_{1}^{h}} \widetilde{\varepsilon}_{3}^{s}\left(x_{1}^{\prime}, k_{2}, \omega\right) e^{j\left(k_{1}^{h}+k_{1}^{s}\right) x_{1}^{\prime}} d x_{1}^{\prime}, \\
\tilde{r}_{2}^{s}\left(k_{2}, \omega\right)=-j k_{0} \frac{t_{2}^{h} t_{2}^{s}}{2 \beta_{2}^{s}} \int_{0}^{x_{1}^{h}}\left[\widetilde{\varepsilon}_{2}^{s}\left(x_{1}^{\prime}, k_{2}, \omega\right)\right. \\
\left.-\frac{k_{2}}{k_{1}^{s}} \widetilde{\varepsilon}_{6}^{s}\left(x_{1}^{\prime}, k_{2}, \omega\right)\right] e^{j\left(k_{1}^{h}+k_{1}^{s}\right) x_{1}^{\prime}} d x_{1}^{\prime} .
\end{gathered}
$$

The meaning of the analytical expressions (23) shall now be commented on. The incident beam $\boldsymbol{E}^{\text {inc }}$ is scattered in the direction $\boldsymbol{k}^{s}=\left(k_{1}^{s}, k_{2}\right)$. The corresponding electric displacement, namely, the optical vibration, becomes ${ }^{(2)} \bar{\varepsilon}^{s} \boldsymbol{E}^{i n c}$. As the material is isotropic, the direction $\boldsymbol{k}^{s}$ must be perpendicular to the direction of scattered polarization $\boldsymbol{P}^{s}=\left(k_{2},-k_{1}^{s}\right)$. To respect this condition, the part of the vibration which is scattered is the projection of the modification of electric vibration ${ }^{(2)} \bar{\varepsilon}^{s} \boldsymbol{E}^{\text {inc }}$ on the direction of scattered polarization $\boldsymbol{P}^{s}$. Therefore, the variation of the scattered amplitude is proportional to the scalar product $\boldsymbol{P}^{s}{ }^{(2)} \bar{\varepsilon}^{s} \boldsymbol{E}^{i n c}$. Thus, as can be seen in expression (23), the beam polarized in the direction $\boldsymbol{x}_{3}$ is sensitive to $\varepsilon_{3}^{s}$, and the one polarized in the plane $\left(\boldsymbol{x}_{1}, \boldsymbol{x}_{2}\right)$ is sensitive to $k_{1}^{s} \varepsilon_{2}^{s}-k_{2} \varepsilon_{6}^{s}$. As a consequence, if the direction of scattered polarization $\boldsymbol{P}^{s}$ is perpendicular to the direction of variation of optical vibration, there is no effect on the reflection coefficient. The beam is only sensitive to the optical variation in the direction of its polarization. For this reason, to be sensitive to shear strain, an oblique acousto-optic interaction is necessary.

To understand the meaning of expressions (23), the dielectric perturbation can be decomposed into a continuous sum of elementary contributions. The acoustic plane wave, while propagating, creates a grating of optical properties, the spacing of which is equal to the acoustic wavelength. It can be represented by a superposition of phase planes, each of them acting as an optical interface localized in $x_{1}^{\prime}$. As plotted in Fig. 1, they backscatter the incident beam of wave vector $\boldsymbol{k}^{h}=\left(k_{1}^{h}, 0\right)$ in the direction defined by $\boldsymbol{k}^{s}=\left(k_{1}^{s}, k_{2}\right)$, for a fixed $k_{2}$ imposed by the spatial Fourier transform. The exponential terms in Eq. (23) describes the phase delay induced by this optical path inside the material. Taking into account the acoustic propagation in both directions $\pm x_{1}$, the components of $\left[\widetilde{\varepsilon}^{s}\right]$ in Eq. (2) are proportional to the term $\exp \left( \pm j k_{1}^{e} x_{1}\right)$. The argument of the exponential is thereby changed to $k_{1}^{h}$ $+k_{1}^{s} \pm k_{1}^{e}$, thus describing the conservation of momentum for a fixed $k_{2} \cdot{ }^{30}$ The acoustic wave propagation favors two scat-
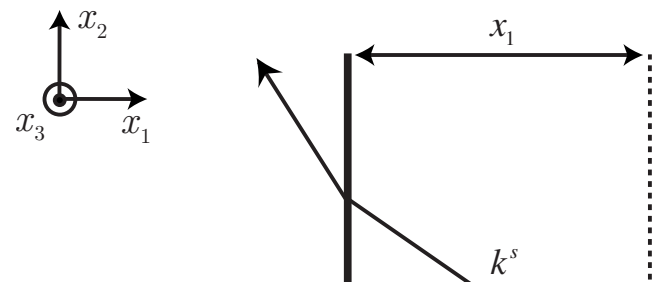

acoustic

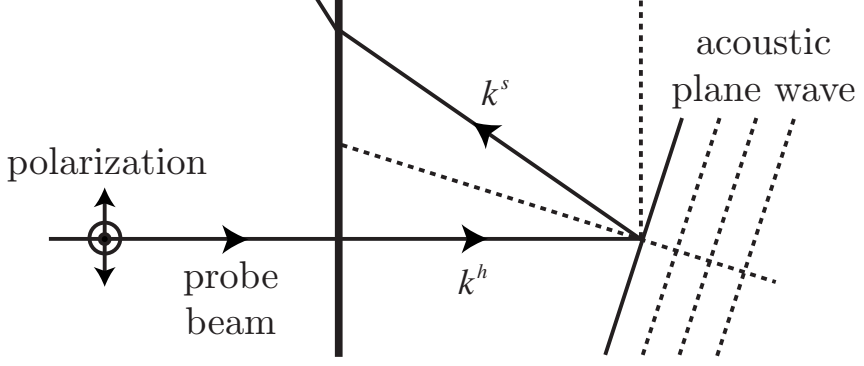

FIG. 1. Interaction of the normal incidence probe beam with a single plane wave propagating in an oblique direction.

tered optical wave vectors ${ }^{+} \boldsymbol{k}^{s}=\boldsymbol{k}^{s}+\boldsymbol{k}^{\boldsymbol{e}}$ and ${ }^{-} \boldsymbol{k}^{s}=\boldsymbol{k}^{\boldsymbol{s}}-\boldsymbol{k}^{\boldsymbol{e}}$. The other terms in Eq. (23), defining the amplitude of the exponentials, are related to the reflection coefficient of the laser beam on the acoustic phase plane. Therefore, they are proportional to $\varepsilon_{3}^{s}$ and $k_{1}^{s} \varepsilon_{2}^{s}-k_{2} \varepsilon_{6}^{s}$, respectively, as explained above.

Finally, the lateral extent of pump and probe is accounted for, multiplying $\left[\widetilde{r}^{s}\right]$ by the spectrum of functions $g_{b}$ and $g_{r}$, respectively. The latter define the spatial distributions along $\boldsymbol{x}_{2}$ of the pump and probe pulses as Gaussian shapes of full width at half maximum (FWHM) $\chi_{b}$ and $\chi_{r}$, respectively. This is equivalent to a single multiplication in the Fourier domain by a Gaussian function of FWHM $\chi^{-1}=\left(\chi_{r}^{2}+\chi_{b}^{2}\right)^{-1 / 2}$. Thus, the smaller the laser spots are, the larger the spectrum in the $k_{2}$ space is, and the higher the sensibility to acoustic diffraction is.

An electric field $\boldsymbol{E}^{i n c}=E^{i n c}\left(\cos \varphi \boldsymbol{x}_{2}+\sin \varphi \boldsymbol{x}_{3}\right)$, polarized in the plane $\left(\boldsymbol{x}_{2}, \boldsymbol{x}_{3}\right)$, is now taken into account. The variable $\varphi$ stands for the angle between the polarization of the probe beam and the axis $\boldsymbol{x}_{2}$. A combination of the previously calculated coefficients is then made to determine the variation of scattered amplitude:

$$
\tilde{r}^{s}=\widetilde{r}_{2}^{s} \cos ^{2} \varphi+\widetilde{r}_{3}^{s} \sin ^{2} \varphi .
$$

As the material is transversally isotropic, changing the orientation of the line source is equivalent to changing the direction of the polarization. Thus, $\varphi$ defines the orientation of the line source for a fixed polarization.

A point source generation is then obtained by applying a Hankel transform to the spectrum of the response to a line source $\widetilde{r}^{s}\left(k_{2}, \omega\right){ }^{19}$ It is equivalent to the summation of line sources of the same Gaussian shape defined by the parameter $\chi$, oriented in different directions $\varphi$. This operation is performed by multiplying the spectrum by Bessel functions ${ }^{31}$ of the first kind $J_{0}$ and $J_{1}$ :

$$
\begin{aligned}
r^{s}\left(x_{2}, \omega\right)= & \int_{-\infty}^{+\infty}\left\{\tilde{r}_{2}^{s}\left[k_{2} J_{0}\left(-k_{2} x_{2}\right)+\frac{J_{1}\left(-k_{2} x_{2}\right)}{x_{2}}\right]\right. \\
& \left.-\tilde{r}_{3}^{s} \frac{J_{1}\left(-k_{2} x_{2}\right)}{x_{2}}\right\} e^{j \omega t} d k_{2} .
\end{aligned}
$$

Finally, to return to the time domain, a numerical inverse Fourier transform is performed over $\omega$. 
$2 \mathrm{~L}$

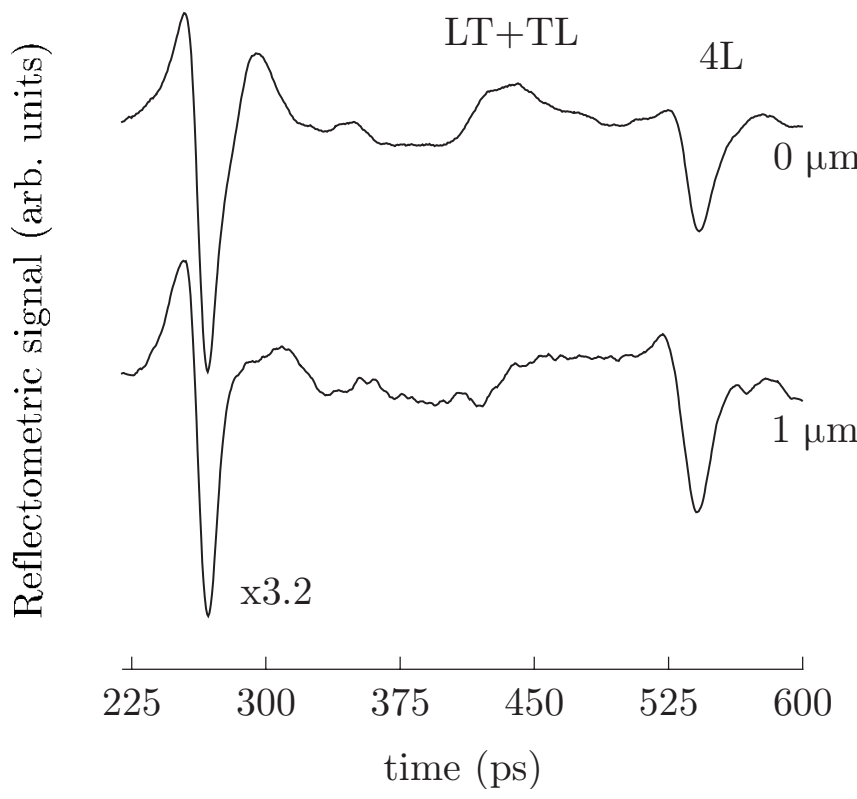

FIG. 2. Measured reflectometric signal in an isotropic aluminum film deposited on Mylar for pump-probe distances of 0 and $1 \mu \mathrm{m}$. Longitudinal waves $2 \mathrm{~L}$ and $4 \mathrm{~L}$, together with the mode converted TL and LT waves, are observed.

\section{THEORETICAL AND EXPERIMENTAL RESULTS}

Experiments are carried out with a pump-probe technique in an isotropic aluminum film of thickness $d=0.9 \mu \mathrm{m}$, deposited on a $3.5 \mu \mathrm{m}$ thick Mylar substrate. Pulses of energy of $5 \mathrm{~nJ}$ and duration of $100 \mathrm{fs}$ are generated by a Ti:sapphire laser, the repetition rate of which is $82 \mathrm{MHz}$. The wavelength is initially $\lambda=796 \mathrm{~nm}$, and the pump beam passes through a doubling crystal to obtain a wavelength of $398 \mathrm{~nm}$, which allows reducing the minimum size of the spot due to diffraction limit. By increasing the probe path, the delay line allows tuning the time difference between the generation and the detection pulses to measure the variation of reflectivity in time. The pump is modulated by an acousto-optic modulator on reference frequency at $300 \mathrm{kHz}$ to detect the signals in the probe by lock-in amplification.

Both beams are focused by a microscope objective to spots on the surface of the film. A $\times 100$ magnification is used with a numerical aperture $\mathrm{NA}=0.8$. As explained in the previous sections, diffracted acoustic waves scatter the reflected light in several space directions. The latter are collected within a certain angle, the size of which must be large in order to collect the scattered light and to be sensitive to acoustic diffraction. To estimate the diameters of the spots on the surface, the space cross correlation of the pump and probe beams is fitted with a Gaussian shape, the FWHM of which is $\chi=1.2 \mu \mathrm{m}$.

As the focal length of the objective is very small, both beams must pass through the same objective. Therefore, a moving lens is used to change slightly the angle of incidence of the pump on the objective and to allow displacing the beam on the surface of the sample. ${ }^{32}$ If pump and probe are not at the same position, acoustic waves propagating in ob-

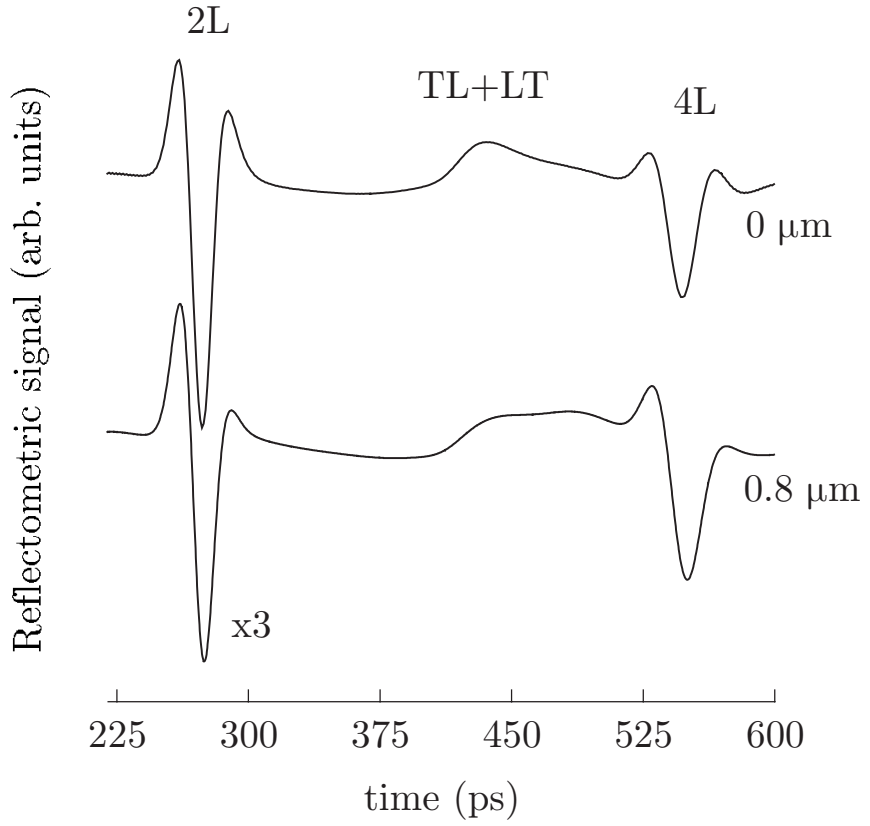

FIG. 3. Calculated reflectometric signal in a free isotropic aluminum film for different pump-probe distances: 0 and $0.8 \mu \mathrm{m}$. Longitudinal waves $2 \mathrm{~L}$ and $4 \mathrm{~L}$, together with the mode converted TL and LT waves, can be observed.

lique directions can be detected. Thus, reflectometric measurements are plotted in Fig. 2 for several pump-probe distances. The signal background, corresponding to the slow thermal relaxation of the material, is removed by subtraction of a polynomial: only the acoustic contribution is represented. The longitudinal echoes which have performed one and two round trips inside the material, namely, $2 \mathrm{~L}$ and $4 \mathrm{~L}$, can be identified from their times of arrival. The periodicity of their apparition matches the ratio $2 d / v_{L} \approx 281 \mathrm{ps}$, with $v_{L} \approx 6.4 \mathrm{~nm} / \mathrm{ps}$ the longitudinal velocity in polycrystalline aluminum. ${ }^{33}$ Between the two latter, superimposed LT and TL waves are observed. Then, the pump-probe distance is increased by moving the lens, and the evolution of the measured signal is discussed below, based on theoretical simulations.

A theoretical calculation is now performed to represent the three-dimensional features of the experiments. As the acoustic impedance of the Mylar substrate is small, a freestanding isotropic aluminum film is modeled, according to the thermal and elastic values given in literature. ${ }^{33,34}$ The strain is calculated, and the reflectometric detection process is described using the matricant for two different pumpprobe distances, as plotted in Fig. 3. The photoelastic coefficients are adjusted to $P_{11}=-10+5 j$ and $P_{12}=-10-j$ to match the experimental results. Their amplitude is arbitrary; only their ratio is relevant. ${ }^{35}$ As the material is isotropic, it is assumed that $P_{66}=\left(P_{11}-P_{12}\right) / 2$.

The longitudinal echoes and the contribution of the transverse echo LT+TL are very well represented, together with their times of arrival. The three polar echoes $2 \mathrm{~L}$ tend to become bipolar as the third pole component is diminishing when increasing pump-probe distance. This change, together with the evolution of the shear wave echo, is accurately de- 
scribed by the calculations. All these features cannot be represented by a one-dimensional model, and demonstrate the necessity to take into account acoustic diffraction in the detection process.

\section{CONCLUSION}

The 3D photoacoustic interaction, occurring during the detection process in picosecond ultrasonics, has been described semianalytically. A convenient method to calculate the electromagnetic field scattered by a medium with continuously varying properties in one direction has been presented. Within the frame of small perturbation theory, it has been applied to a tensorial variation of the optical index induced by the propagation of a three-dimensional acoustic field.

The small lateral size of the laser source allows generation of an intricate acoustic field, including mode conversion and shear wave propagation. These effects modify the dielectric properties through the elasto-optic interaction, deforming and rotating the surface of optical indices. The linearization of this coupling, in the direction perpendicular to the orientation of the line source on the surface, suppresses the spectral convolution in the source term of Maxwell equations. Thus, the change of reflectivity can be expressed analytically in the Fourier domain using the matricant, and the mechanisms of acousto-optic interaction are explained, since they now appear explicitly in three-dimensional configurations. The probe beam is only sensitive to the variation of optical properties in the direction of its polarization. As a consequence, the detection of shear plane wave requires an oblique interaction between the acoustic plane wave and the optical beam. In the present case, this condition is fulfilled by the acoustic diffraction. Finally, a Hankel transform is applied to calculate the response to a point source.

Good agreement is found between theoretical results and reflectometric measurements. Shear wave detection, together with the effect of acoustic diffraction on the shape of the echoes, is matched. The elasto-optic interaction modeling introduced in this paper could be applied to the characterization of multilayered structures in picosecond ultrasonics, considering the succession of layers as a discrete change in properties. Besides, the propagation of longitudinal and shear waves, together with Rayleigh waves, is included in the so- lution of the equation of motion. Therefore, the acousto-optic detection of surface waves, using reflectometric measurements, could be studied in the frame of the proposed model.

\section{ACKNOWLEDGMENTS}

The authors acknowledge helpful discussions with A. Shuvalov on the mathematical aspects of the matricant. This work is partially supported by a grant from the regional council of Aquitaine, a grant from the "Agence Nationale de la Recherche" within the PNANO program, and the European Union.

\section{APPENDIX: COEFFICIENTS OF THE EXPANSION} MATRIX

$$
\begin{aligned}
& m_{11}=\frac{k_{0}}{\beta_{3}^{s}} \int_{0}^{x_{1}} a_{21} s_{1}^{s} c_{1}^{h} d x_{1}^{\prime}, \\
& m_{12}=\frac{j k_{0}}{\beta_{3}^{h} \beta_{3}^{s}} \int_{0}^{x_{1}} a_{21} s_{1}^{h} s_{1}^{s} d x_{1}^{\prime}, \\
& m_{21}=j k_{0} \int_{0}^{x_{1}} a_{21} c_{1}^{h} c_{1}^{s} d x_{1}^{\prime}, \\
& m_{22}=-\frac{k_{0}}{\beta_{3}^{h}} \int_{0}^{x_{1}} a_{21} s_{1}^{h} c_{1}^{s} d x_{1}^{\prime}, \\
& m_{33}=j k_{0} \int_{0}^{x_{1}} a_{33} c_{1}^{h} c_{1}^{s} d x_{1}^{\prime}+\frac{k_{0}}{\beta_{2}^{s}} \int_{0}^{x_{1}} a_{43} c_{1}^{h} s_{1}^{s} d x_{1}^{\prime}, \\
& m_{44}=\frac{j k_{0} \beta_{2}^{s}}{\beta_{2}^{h}} \int_{0}^{x_{1}} a_{33} s_{1}^{h} s_{1}^{s} d x_{1}^{\prime}-\frac{k_{0}}{\beta_{2}^{h}} \int_{0}^{x_{1}} a_{43} s_{1}^{h} c_{1}^{s} d x_{1}^{\prime}, \\
& m_{34}=\frac{j k_{0}}{\beta_{2}^{h} \beta_{2}^{s}} \int_{0}^{x_{1}} a_{43} s_{1}^{h} s_{1}^{s} d x_{1}^{\prime}-\frac{k_{0}}{\beta_{2}^{h}} \int_{0}^{x_{1}} a_{33} s_{1}^{h} c_{1}^{s} d x_{1}^{\prime}, \\
& k_{2}^{s} a_{33}^{x_{1}} c_{1}^{h} s_{1}^{s} d x_{1}^{\prime}+j k_{0} \int_{0}^{x_{1}} a_{43} c_{1}^{h} c_{1}^{s} d x_{1}^{\prime},
\end{aligned}
$$

with $s_{1}^{q}=\sin \left(k_{1}^{q} x_{1}^{\prime}\right)$ and $c_{1}^{q}=\cos \left(k_{1}^{q} x_{1}\right)$. *b.audoin@1mp.u-bordeaux1.fr

${ }^{1}$ C. Thomsen, J. Strait, Z. Vardeny, H. J. Maris, J. Tauc, and J. J. Hauser, Phys. Rev. Lett. 53, 989 (1984).

${ }^{2}$ C. Thomsen, H. T. Grahn, H. J. Maris, and J. Tauc, Opt. Commun. 60, 55 (1986).

${ }^{3}$ L. D. Landau and E. M. Lifchitz, Theory of Elasticity (Mir, Moscow, 1967).

${ }^{4}$ D. H. Hurley, O. B. Wright, O. Matsuda, V. E. Gusev, and O. V. Kolosov, Ultrasonics 38, 470 (2000).

${ }^{5}$ T. Bienville and B. Perrin, Proceedings of the WCU, Paris, 2003 (unpublished), p. 813.

${ }^{6}$ O. Matsuda, O. B. Wright, D. H. Hurley, V. E. Gusev, and K. Shimizu, Phys. Rev. Lett. 93, 095501 (2004).

${ }^{7}$ T. Pezeril, N. Chigarev, P. Ruello, S. Gougeon, D. Mounier, J.-M. Breteau, P. Picart, and V. E. Gusev, Phys. Rev. B 73, 132301 (2006).

${ }^{8}$ P. Cielo, F. Nadeau, and M. Lamontagne, Ultrasonics 23, 55 (1985).

${ }^{9}$ A. Neubrand and P. Hess, J. Appl. Phys. 71, 227 (1992).

${ }^{10}$ A. A. Maznev, A. Akthakul, and K. A. Nelson, J. Appl. Phys. 86, 
2818 (1999).

${ }^{11}$ Y. Sugawara, O. B. Wright, O. Matsuda, M. Takigahira, Y. Tanaka, S. Tamura, and V. E. Gusev, Phys. Rev. Lett. 88, 185504 (2002).

${ }^{12}$ H. Yamazaki, O. Matsuda, and O. B. Wright, Phys. Status Solidi C 1, 2991 (2004).

${ }^{13}$ N. C. R. Holme, B. C. Daly, M. T. Myaing, and T. B. Norris, Appl. Phys. Lett. 83, 392 (2003).

${ }^{14}$ B. C. Daly, T. B. Norris, J. Chen, and J. B. Khurgin, Phys. Rev. B 70, 214307 (2004).

${ }^{15}$ C. Rossignol, J. M. Rampnoux, M. Perton, B. Audoin, and S. Dilhaire, Phys. Rev. Lett. 94, 166106 (2005).

${ }^{16}$ B. Perrin, C. Rossignol, B. Bonello, and J. C. Jeannet, Physica B 263-264, 571 (1999).

${ }^{17}$ R. Weaver, W. Sachse, and K. Kim, J. Appl. Mech. 63, 338 (1996).

${ }^{18}$ B. Audoin, H. Meri, and C. Rossignol, Phys. Rev. B 74, 214304 (2006).

${ }^{19}$ M. Perton, Ph.D. thesis, Université Bordeaux 1, 2006.

${ }^{20}$ D. F. Nelson and M. Lax, Phys. Rev. B 3, 2778 (1971).

${ }^{21}$ A. Miklós and A. Lörincz, Appl. Phys. B: Photophys. Laser Chem. 48, 261 (1989).

${ }^{22}$ M. Born and E. Wolf, Principles of Optics (Cambridge University Press, Cambridge, 1999).
${ }^{23}$ O. Matsuda and O. B. Wright, Ultrasonics 42, 653 (2004).

${ }^{24}$ M. C. Pease, Methods of Matrix Algebra (Academic, New York, 1965).

${ }^{25}$ C. Baron, A. L. Shuvalov, and O. Poncelet, Ultrasonics 46, 1 (2007).

${ }^{26}$ C. V. Shank and E. P. Ippen, Appl. Phys. Lett. 24, 373 (1974).

${ }^{27} \mathrm{O}$. Wright and V. Gusev, IEEE Trans. Ultrason. Ferroelectr. Freq. Control 42, 331 (1995).

${ }^{28}$ J. H. Bechtel, J. Appl. Phys. 46, 1585 (1975).

${ }^{29}$ B. Auld, Acoustic Fields and Waves in Solids (Krierger, Malabar, FL, 1990), Vol. 1.

${ }^{30}$ C. Kittel, Solid State Physics, 7th ed. (Wiley Interscience, New York, 1995).

${ }^{31} \mathrm{G}$. N. Watson, A Treatrise on the Theory of Bessel Functions (Cambridge University Press, Cambridge, 1966).

${ }^{32}$ M. Perton, C. Rossignol, N. Chigarev, and B. Audoin, in Quantitative Nondestructive Evaluation, edited by D. O. Thompson and D. E. Chimenti, AIP Conf. Proc. No. 26 (AIP, New York, 2007).

${ }^{33}$ O. L. Anderson, Physical Acoustics (Masson, Paris/Academic, New York, 1965), Vol. 3B.

${ }^{34}$ D. Lide, CRC Handbook of Chemistry and Physics (CRC, Boca Raton, FL, 2002).

${ }^{35}$ A. Devos and C. Lerouge, Phys. Rev. Lett. 86, 2669 (2001). 